\title{
Estimate of Exponential Convergence Rate and Exponential Stability for Neural Networks
}

\author{
Zhang Yi, P. A. Heng, and Ada W. C. Fu
}

\begin{abstract}
Estimate of exponential convergence rate and exponential stability are studied for a class of neural networks which includes the Hopfield neural networks and the cellular neural networks. Both local and global exponential convergence is discussed. Theorems for estimate of exponential convergence rate are established and the bounds on the rate of convergence are given. The domains of attraction in the case of local exponential convergence are obtained. Simple conditions are presented for checking exponential stability of the neural networks.
\end{abstract}

Index Terms-Convergence rate, neural networks, stability.

\section{INTRODUCTION}

$\mathbf{W}^{\mathrm{s}}$ E shall consider in this paper the neural networks described by the following nonlinear differential equation:

$$
\begin{gathered}
\frac{d x_{i}(t)}{d t}=-d_{i} x_{i}(t)+\sum_{j=1}^{n} T_{i j} g_{j}\left(x_{j}(t)\right)+I_{i}, \\
t \geq 0, \quad(i=1, \cdots, n)
\end{gathered}
$$

where $\left(x_{1}, \cdots, x_{n}\right)^{T} \in R^{n}, d_{i}>0$, and $I_{i}(i=1, \cdots, n)$ are constants, $\left(T_{i j}\right)_{n \times n}$ is a constant matrix and is referred to as the interconnection matrix, and $g_{i}: R \rightarrow R(i=$ $1, \cdots, n)$ represent the neuron input output activations and are locally Lipschitz continuous functions with $D^{+} g_{i}(s) \geq$ $0(i=1, \cdots, n)$ for all $s \in R$. The symbol $D^{+}$denotes the Dini derivative. For any continuous function $f: R \rightarrow R$ the Dini derivative of $f(t)$ is defined as

$$
D^{+} f(t)=\lim _{h \rightarrow 0^{+}} \sup \frac{f(t+h)-f(t)}{h} .
$$

It is easy to see that if $f(t)$ is locally Lipschitz then $\left|D^{+} f(t)\right|<+\infty$.

Neural networks as (1) have been proposed in [5]. The existence and uniqueness about equilibrium points and the global asymptotic stability of (1) were studied in [5] and [6]. From the mathematical point of view, this kind of neural networks includes the Hopfield neural networks [9] and the cellular neural networks [2]. In fact if the neural activations satisfy $0<D^{+} g_{i}(s) \leq k_{i}(i=1, \cdots, n)$ then (1) describes the Hopfield neural networks. If $g_{i}(s)=(|s+1|+|s-1|) / 2$, $(i=1, \cdots, n)$ then (1) describes the cellular neural networks.

So far, the continuous models of neural networks such as Hopfield neural networks and cellular neural networks have got many successful applications, for example, in the application of solving some optimization problems and associative

Manuscript received May 7, 1998; revised September 11, 1998 and May 27, 1999. This work was supported in part by RGC Earmarked Grant CUHK 4306/98E of Hong Kong and the National Science Foundation of China under Grant 69871005.

The authors are with the Department of Computer Science and Engineering, The Chinese University of Hong Kong, Hong Kong.

Publisher Item Identifier S 1045-9227(99)08832-3. memory. In most of the applications of neural networks there is a shared requirement of raising the networks convergence speed in order to cut down the time of neural computing. Since the exponential convergence rate could be used to determine the speed of neural computation, it is interesting to study the estimate of exponential convergence rate and exponential stability of neural networks. Some results on the exponential stability of neural networks could be found in [1], [8], and [10]. In this paper we shall study the estimation of exponential convergence rate and the exponential stability of (1). Both local and global exponential convergence will be discussed. This paper is organized as follows. Global exponential convergence analysis will be given in Section II. Local exponential convergence analysis will be given in Section III. Examples in Section IV will be employed to illustrate the theory.

\section{Global EXPonential Convergence AnAlysis}

If neural networks are employed to solve some optimization problems by using the related energy functions, it is desired to design a neural network with less equilibria. In fact, if a neural network has a unique global convergence equilibrium, it will be the global minimum point of the related energy function. Since the global stability of a neural network implies that the neural network has a unique equilibrium and all solutions of the network converge to the equilibrium, global stability analysis is interesting in the application of solving optimization problems. For the Hopfield neural networks some elegant conditions of global asymptotic stability were reported in [3]. These conditions are weaker than many existing global asymptotic stability conditions of Hopfield neural networks and they are easy to check in practice. In [5], the global asymptotic stability conditions in [3] were generalized to the more general neural networks of (1). Some unnecessary conditions of [5] were omitted in [6]. In the present section, we shall establish the estimate of global exponential convergence rate of (1). The bounds on the rate of global exponential convergence will be given. Moreover, we shall show that similar conditions for global asymptotic stability in [5], [6] imply global exponential stability. It should be noticed that since the neural network of (1) is described by nonlinear ordinary differential equation, the global asymptotic stability does not imply global exponential stability automatically. There are many nonlinear differential systems that are globally asymptotically stable but not globally exponentially stable.

Throughout this section we assume that there exist some constants $k_{i}>0(i=1, \cdots, n)$ such that

$$
0 \leq D^{+} g_{i}(s) \leq k_{i} \quad(i=1, \cdots, n)
$$

for all $s \in R$. 
Next, we prove an inequality for the neuron input output activations that will be used to study the global exponential convergence of (1).

Lemma 1: We have

$$
\begin{aligned}
\int_{v}^{u} & {\left[g_{i}(s)-g_{i}(v)\right] d s } \\
& \geq \frac{1}{2 k_{i}}\left[g_{i}(u)-g_{i}(v)\right]^{2} \quad(i=1, \cdots, n)
\end{aligned}
$$

for all $u, v \in R$.

Proof: Define continuous functions

$$
\begin{aligned}
E_{i}(u)=\int_{v}^{u} & {\left[g_{i}(s)-g_{i}(v)\right] d s-\frac{1}{2 k_{i}}\left[g_{i}(u)-g_{i}(v)\right]^{2} } \\
& (i=1, \cdots, n) .
\end{aligned}
$$

Then it follows that

$$
D^{+} E_{i}(u)=\left[g_{i}(u)-g_{i}(v)\right]\left[1-\frac{D^{+} g_{i}(u)}{k_{i}}\right] .
$$

Since $0 \leq D^{+} g_{i}(s) \leq k_{i}$ for all $s \in R$, we have

$$
D^{+} E_{i}(u) \begin{cases}\geq 0, & u>v \\ \leq 0, & u<v \\ =0, & u=v .\end{cases}
$$

This shows that $u=v$ is the minimum point of the function $E_{i}(u)$ and so it follows for any $u \in R$ that $E_{i}(u) \geq E_{i}(v)=$ 0 . Hence

$$
\begin{aligned}
\int_{v}^{u} & {\left[g_{i}(s)-g_{i}(v)\right] d s } \\
& \geq \frac{1}{2 k_{i}}\left[g_{i}(u)-g_{i}(v)\right]^{2} \quad(i=1, \cdots, n) .
\end{aligned}
$$

The proof is completed.

Noting that $0 \leq D^{+} g_{i}(s) \leq k_{i}$ implies that

$$
0 \leq \frac{g_{i}\left(\xi_{i}\right)-g_{i}\left(\xi_{2}\right)}{\xi_{1}-\xi_{2}} \leq k_{i}
$$

for each $\xi_{1}, \xi_{2} \in R, \xi_{1} \neq \xi_{2}$, the following Lemma about the existence and uniqueness of equilibrium points is adopted from [5] and [6].

Lemma 2: If there exist constants $\alpha_{i}>0(i=1, \cdots, n)$ such that the matrix

$$
\left[-\frac{\alpha_{i} d_{i}}{k_{i}} \delta_{i j}+\frac{\alpha_{i} T_{i j}+\alpha_{j} T_{j i}}{2}\right]_{n \times n}, \quad \delta_{i j}= \begin{cases}1, & i=j \\ 0, & i \neq j\end{cases}
$$

is negative definite, then the neural network of (1) has a unique equilibrium point.

We say that an equilibrium $\left(x_{1}^{*}, \cdots, x_{n}^{*}\right)^{T}$ of (1) is globally exponentially stable if there is a $\epsilon>0$ and some $M \geq 1$ such that

$$
\sum_{i=1}^{n}\left|x_{i}(t)-x_{i}^{*}\right| \leq M|| x(0)-x^{*}|| e^{-\epsilon t}
$$

for all $t \geq 0$, where

$$
\left\|x(0)-x^{*}\right\|=\max _{1 \leq i \leq n}\left(\left|x_{i}(0)-x_{i}^{*}\right|\right) .
$$

Theorem 1: If there exists $\sigma \in\left(0, \min _{1 \leq i \leq n}\left(d_{i}\right)\right)$ such that the matrix

$$
T_{\sigma}=\left[-\frac{d_{i}-\sigma}{k_{i}} \delta_{i j}+\frac{T_{i j}+T_{j i}}{2}\right]_{n \times n}, \quad \delta_{i j}= \begin{cases}1, & i=j \\ 0, & i \neq j\end{cases}
$$

is negative semidefinite, then (1) has an unique equilibrium point $\left(x_{1}^{*}, \cdots, x_{n}^{*}\right)^{T}$ and the solution $\left(x_{1}(t), \cdots, x_{n}(t)\right)^{T}$ starting from any point $\left(x_{1}(0), \cdots, x_{n}(0)\right)^{T} \in R^{n}$ satisfies

$$
\begin{aligned}
\left|x_{i}(t)-x_{i}^{*}\right| \leq \| x(0)-x^{*}|| & e^{-d_{i} t}+\frac{2}{2 d_{i}-\sigma} \sum_{j=1}^{n} \\
& \cdot\left|T_{i j}\right| \sqrt{\left.2 k_{j} \sum_{r=1}^{n} k_{r} e^{-(\sigma / 2) t}\right]}
\end{aligned}
$$

for all $t \geq 0,(i=1, \cdots, n)$.

Proof: Since the matrix $T_{\sigma}$ is negative semidefinite implies that the matrix

$$
\left[-\frac{d_{i}}{k_{i}} \delta_{i j}+\frac{T_{i j}+T_{j i}}{2}\right]_{n \times n}
$$

is negative definite, then by Lemma 2, (1) has a unique equilibrium point $\left(x_{1}^{*}, \cdots, x_{n}^{*}\right)^{T}$. Define a function

$$
V(t)=\sum_{i=1}^{n} \int_{x_{i}^{*}}^{x_{i}(t)}\left[g_{i}(\theta)-g_{i}\left(x_{i}^{*}\right)\right] d \theta
$$

for all $t \geq 0$. Obviously, $V(t) \geq 0$. Computing the time derivative of $V(t)$ it follows that

$$
\begin{aligned}
\frac{d V(t)}{d t}= & -\sum_{i=1}^{n} d_{i}\left[g_{i}\left(x_{i}(t)\right)-g_{i}\left(x_{i}^{*}\right)\right]\left(x_{i}(t)-x_{i}^{*}\right) \\
& +\sum_{i=1}^{n} \sum_{j=1}^{n} T_{i j}\left[g_{i}\left(x_{i}(t)\right)-g_{i}\left(x_{i}^{*}\right)\right] \\
& \cdot\left[g_{j}\left(x_{j}(t)\right)-g_{j}\left(x_{j}^{*}\right)\right] \\
= & -\sigma \sum_{i=1}^{n}\left[g_{i}\left(x_{i}(t)\right)-g_{i}\left(x_{i}^{*}\right)\right]\left(x_{i}(t)-x_{i}^{*}\right) \\
& -\sum_{i=1}^{n}\left(d_{i}-\sigma\right)\left[g_{i}\left(x_{i}(t)\right)-g_{i}\left(x_{i}^{*}\right)\right]\left(x_{i}(t)-x_{i}^{*}\right) \\
& +\sum_{i=1}^{n} \sum_{j=1}^{n} \frac{T_{i j}+T_{j i}}{2}\left[g_{i}\left(x_{i}(t)\right)-g_{i}\left(x_{i}^{*}\right)\right] \\
& \cdot\left[g_{j}\left(x_{j}(t)\right)-g_{j}\left(x_{j}^{*}\right)\right] \\
\leq & -\sigma \sum_{i=1}^{n}\left[g_{i}\left(x_{i}(t)\right)-g_{i}\left(x_{i}^{*}\right)\right]\left[x_{i}(t)-x_{i}^{*}\right) \\
& +\sum_{i=1}^{n} \sum_{j=1}^{n}\left[-\frac{d_{i}-\sigma}{k_{i}} \delta_{i j}+\frac{T_{i j}+T_{j i}}{2}\right] \\
& \cdot\left[g_{i}\left(x_{i}(t)\right)-g_{i}\left(x_{i}^{*}\right)\right]\left[g_{j}\left(x_{j}(t)\right)-g_{j}\left(x_{j}^{*}\right)\right]
\end{aligned}
$$


for all $t \geq 0$. Since $T_{\sigma}$ is semidefinite, then

$$
\begin{aligned}
\frac{d V(t)}{d t} & \leq-\sigma \sum_{i=1}^{n}\left[g_{i}\left(x_{i}(t)\right)-g_{i}\left(x_{i}^{*}\right)\right]\left(x_{i}(t)-x_{i}^{*}\right) \\
& \leq-\sigma \sum_{i=1}^{n} \int_{x_{i}^{*}}^{x_{i}(t)}\left[g_{i}(\theta)-g_{i}\left(x_{i}^{*}\right)\right] d \theta \\
& =-\sigma V(t)
\end{aligned}
$$

and so $V(t) \leq V(0) e^{-\sigma t}$ for all $t \geq 0$. It follows from (2) that

$$
\begin{aligned}
V(0) & =\sum_{i=1}^{n} \int_{x_{i}^{*}}^{x_{i}(0)}\left[g_{i}(\theta)-g_{i}\left(x_{i}^{*}\right)\right] d \theta \\
& \leq \sum_{i=1}^{n}\left[g_{i}\left(x_{i}(0)\right)-g_{i}\left(x_{i}^{*}\right)\right]\left[x_{i}(0)-x_{i}^{*}\right] \\
& \leq \sum_{i=1}^{n} k_{i}\left[x_{i}(0)-x_{i}^{*}\right]^{2} \\
& \leq\left\|x(0)-x^{*}\right\|^{2} \sum_{i=1}^{n} k_{i}
\end{aligned}
$$

and by Lemma 1

$$
\begin{aligned}
V(t) & \geq \int_{x_{i}^{*}}^{x_{i}(t)}\left[g_{i}(\theta)-g_{i}\left(x_{i}^{*}\right)\right] d \theta \\
& \geq \frac{1}{2 k}\left[g_{i}\left(x_{i}(t)\right)-g_{i}\left(x_{i}^{*}\right)\right]^{2}, \quad(i=1, \cdots, n)
\end{aligned}
$$

for all $t \geq 0$, then we have

$$
\begin{gathered}
\left|g_{i}\left(x_{i}(t)\right)-g_{i}\left(x_{i}^{*}\right)\right| \\
\leq\left\|x(0)-x^{*}\right\| \sqrt{2 k_{i} \sum_{j=1}^{n} k_{j} e^{-(\sigma / 2) t}} \\
(i=1, \cdots, n)
\end{gathered}
$$

for all $t \geq 0$.

From (1) it follows that

$$
\begin{aligned}
\frac{d\left[x_{i}(t)-x_{i}^{*}\right]}{d t}= & -d_{i}\left[x_{i}(t)-x_{i}^{*}\right] \\
& +\sum_{j=1}^{n} T_{i j}\left[g_{j}\left(x_{j}(t)\right)-g_{j}\left(x_{j}^{*}\right)\right]
\end{aligned}
$$

for all $t \geq 0$, then by (4) we have

$$
\begin{aligned}
D^{+}\left|x_{i}(t)-x_{i}^{*}\right| & \\
\leq & -d_{i}\left|x_{i}(t)-x_{i}^{*}\right|+\sum_{j=1}^{n}\left|T_{i j}\right|\left|g_{j}\left(x_{j}(t)\right)-g_{j}\left(x_{j}^{*}\right)\right| \\
\leq & -d_{i}\left|x_{i}(t)-x_{i}^{*}\right|+\left\|x(0)-x^{*}\right\| \\
& \cdot \| \sum_{j=1}^{n}\left|T_{i j}\right| \sqrt{2 k_{j} \sum_{r=1}^{n} k_{r} e^{-(\sigma / 2) t}}
\end{aligned}
$$

and so

$$
\begin{aligned}
&\left|x_{i}(t)-x_{i}^{*}\right| \leq\left|x_{i}(0)-x_{i}^{*}\right| e^{-d_{i} t}+\left\|x(0)-x^{*}\right\| \\
& \cdot \sum_{j=1}^{n}\left|T_{i j}\right| \sqrt{2 k_{j} \sum_{r=1}^{n} k_{r}} \\
& \cdot \int_{0}^{t} e^{-d_{i}(t-2)} e^{-(\sigma / 2) s} d s \\
& \leq\left\|x(0)-x^{*}\right\|\left[e^{-d_{i} t}+\frac{2}{2 d_{i}-\sigma} \sum_{j=1}^{n}\left|T_{i j}\right|\right. \\
&\left.\cdot \sqrt{2 k_{j} \sum_{r=1}^{n} k_{r}} e^{-(\sigma / 2) t}\right]
\end{aligned}
$$

for all $t \geq 0$. This completes the proof.

In the proof of Theorem 1, we introduced a differentiable function $V(t)$ of (2) to study the exponential convergence of (1). Obviously, since $D^{+} g_{i}(s)(i=1, \cdots, n)$ may take zeroes it results that $V(t)$ is not a Lyapunov function. This is different from the Lyapunov function introduced in [3]. In [3], only sigmoid (bounded and strictly increasing) activations of neural networks were considered. To cover the case of unbounded activations and/or those with infinite intervals with zero slope, [5], [6] used Lyapunov functions of the Lur'e-Postnikov type to study global stability of (1). The Lyapunov functions of the Lur'e-Postnikov type was also used in [4] for studying the global absolute stability of neural networks. However, it seems that the methods of [3]-[6] are difficult to be developed to study the exponential convergence of (1). Indeed, Lemma 1 plays an essential role in the proof of Theorem 1 .

Theorem 2: If the matrix

$$
\left[-\frac{d_{i}}{k_{i}} \delta_{i j}+\frac{T_{i j}+T_{j i}}{2}\right]_{n \times n}, \quad \delta_{i j}= \begin{cases}1, & i=j \\ 0, & i \neq j\end{cases}
$$

is negative definite, then the neural network of (1) has an unique equilibrium and this equilibrium is globally exponentially stable.

Proof: Since the matrix

$$
\left[-\frac{d_{i}}{k_{i}} \delta_{i j}+\frac{T_{i j}+T_{j i}}{2}\right]_{n \times n}
$$

is negative definite, there must exist $\sigma \in\left(0, \min _{1 \leq i \leq n}\left(d_{i}\right)\right)$ such that the matrix

$$
T_{\sigma}=\left[-\frac{d_{i}-\sigma}{k_{i}} \delta_{i j}+\frac{T_{i j}+T_{j i}}{2}\right]_{n \times n}
$$

is negative semidefinite. The result now follows from Theorem 1 and the proof is completed.

To make the above theorems more flexible, we may modify the function (2) as

$$
V(t)=\sum_{i=1}^{n} \alpha_{i} \int_{x_{i}^{*}}^{x_{i}(t)}\left[f(\theta)-f\left(x_{i}^{*}\right)\right] d \theta
$$

where $\alpha_{i}>0(i=1, \cdots, n)$ are some constants. Then we have the following results. 
Theorem 3: If there exist constants $\alpha_{i}>0(i=1, \cdots, n)$ and $0<\sigma<\min _{1 \leq j \leq n}\left(\alpha_{j} d_{j}\right)$ such that the matrix

$$
\left[-\frac{\alpha_{i} d_{i}-\sigma}{k_{i}} \delta_{i j}+\frac{\alpha_{i} T_{i j}+\alpha_{j} T_{j i}}{2}\right]_{n \times n}, \quad \delta_{i j}= \begin{cases}1, & i=j \\ 0, & i \neq j\end{cases}
$$

is negative semidefinite, then (1) has an unique equilibrium point $\left(x_{1}^{*}, \cdots, x_{n}^{*}\right)$ and

$$
\begin{aligned}
\left|x_{i}(t)-x_{i}^{*}\right| \leq & \left\|x(0)-x^{*}\right\| \\
& \cdot\left[e^{-d_{i} t}+\frac{2 \max _{1 \leq r \leq}\left(\alpha_{r}\right)}{2 d_{i} \max _{1 \leq r \leq n}\left(\alpha_{r}\right)-\sigma} \sum_{j=1}^{n}\left|T_{i j}\right|\right. \\
& \cdot \sqrt{\left.\frac{2 k_{j}}{\alpha_{j}} \sum_{r=1}^{n} \alpha_{r} k_{r} e^{-\left(\sigma / 2 \max _{1 \leq r \leq n}\left(\alpha_{r}\right)\right) / t}\right]}
\end{aligned}
$$

for all $t \geq 0,(i=1, \cdots, n)$.

Theorem 4: If there exist constant $\alpha_{i}>0(i=1, \cdots, n)$ such that

$$
\left[-\frac{\alpha_{i} d_{i}}{k_{i}} \delta_{i j}+\frac{\alpha_{i} T_{i j}+\alpha_{j} T_{j i}}{2}\right]_{n \times n}, \quad \delta_{i j}= \begin{cases}1, & =j \\ 0, & i \neq j\end{cases}
$$

is negative definite, then (1) has a unique equilibrium and this equilibrium is globally exponentially stable.

\section{Local Exponential CONVERGENCE ANALYsis}

It is well known that in the application of a neural network to associative memory, the neural network should have more equilibrium than one. Since the global convergence implies that the neural network has a unique equilibrium, the results of last section are not useful in the application of associative memory. In this section we shall develop some qualitative results on the estimate of local exponential convergence rate and local exponential stability. Bounds on convergence rate together with the domain of attraction will be given. By local exponential convergence, it allows the neural network of (1) to store more local exponential stable equilibrium than one.

An equilibrium $\left(x_{1}^{*}, \cdots, x_{n}^{*}\right)^{T}$ of (1) is said to be locally exponentially stable if there is a $\lambda>0$ and for any $\epsilon>0$ there is a $\delta>0$ such that $\left|x_{i}(0)-x_{i}^{*}\right|<\delta(i=1, \cdots, n)$ implies that $\left|x_{i}(t)-x_{i}^{*}\right| \leq \epsilon e^{-\lambda t}(i=1, \cdots, n)$ for all $t \geq 0$.

Lemma 3: Let $D \subseteq R^{n}$ be a convex subset and $\left(x_{1}^{*}, \cdots, x_{n}^{*}\right)^{T} \in D$ be a fixed point. If there exist constants $k_{i}>0(i=1, \cdots, n)$ such that

$$
0 \leq D^{+} g_{i}\left(x_{i}\right) \leq k_{i} \quad(i=1, \cdots, n)
$$

for each $\left(x_{1}, \cdots, x_{n}\right)^{T} \in D$, then

$$
\begin{aligned}
\int_{x_{i}^{*}}^{x_{i}} & {\left[g_{i}(s)-g_{i}\left(x_{i}^{*}\right)\right] d s } \\
& \geq \frac{1}{2 k_{i}}\left[g_{i}\left(x_{i}\right)-g_{i}\left(x_{i}^{*}\right)\right]^{2} \quad(i=1, \cdots, n)
\end{aligned}
$$

for all $\left(x_{1}, \cdots, x_{n}\right)^{T} \in D$.

The proof of Lemma 3 is similar to that of Lemma 1, the details are omitted.
Theorem 5: Let $\left(x_{1}^{*}, \cdots, x_{n}^{*}\right)^{T}$ be equilibrium of (1) in the close set

$$
D=\left\{\left(x_{1}, \cdots, x_{n}\right)^{T}|| x_{i}-x_{i}^{*} \mid \leq \alpha \quad(i=1, \cdots, n)\right\}
$$

where $\alpha>0$ is a constant. Suppose that there exist constants $k_{i}>0(i=1, \cdots, n)$ such that

$$
0 \leq D^{+} g_{i}\left(x_{i}\right) \leq k_{i} \quad(i=1, \cdots, n)
$$

for any $\left(x_{1}, \cdots, x_{n}\right)^{T}$. If there exist $\sigma \in\left(0, \min _{1 \leq i \leq n}\left(d_{i}\right)\right)$ such that the matrix

$$
T_{\sigma}=\left[-\frac{d_{i}-\sigma}{k_{i}} \delta_{i j}+\frac{T_{i j}+T_{j i}}{2}\right]_{n \times n}, \quad \delta_{i j}= \begin{cases}1, & i=j \\ 0, & i \neq j\end{cases}
$$

is negative semidefinite, then the solution $\left(x_{1}(t), \cdots, x_{n}(t)\right)^{T}$ starting from any point $\left(x_{1}(0), \cdots, x_{n}(0)\right)^{T} \in Q$, where

$$
Q=\left\{\left(x_{1}, \cdots, x_{n}\right)^{T}|| x_{i}-x_{i}^{*} \mid<\frac{\alpha}{\beta},(i=1, \cdots, n)\right\} \subset D
$$

and

$$
\beta=\max _{1 \leq i \leq n}\left(1+\frac{2}{2 d_{i}-\sigma} \sum_{j=1}^{n}\left|T_{i j}\right| \sqrt{2 k_{j} \sum_{r=1}^{n} k_{r}}\right)
$$

stays in $D$ and satisfies

$$
\begin{gathered}
\left|x_{i}(t)-x_{i}^{*}\right| \leq|| x(0)-x^{*}||\left[e^{-d_{i} t}+\frac{2}{2 d_{i}-\sigma} \sum_{j=1}^{n}\left|T_{i j}\right|\right. \\
\cdot \sqrt{\left.2 k_{j} \sum_{r=1}^{n} k_{r} e^{-(\sigma / 2) t}\right]}
\end{gathered}
$$

for all $t \geq 0,(i=1, \cdots, n)$.

Proof: We first prove that the solution $\left(x_{1}(t), \cdots, x_{n}(t)\right)^{T} \quad$ starting from any point $\left(x_{1}(0), \cdots, x_{n}(0)\right)^{T}$ of $Q$ stays in $D$ for all $t \geq 0$ and never across the boundary of $D$. Otherwise, since $\left(x_{1}(0), \cdots, x_{n}(0)\right)^{T} \in Q$, there must exist $t_{1}>0$ and some $i$ such that $\left|x_{i}\left(t_{1}\right)-x_{i}^{*}\right|=\alpha$ and $\left|x_{j}(t)-x_{j}^{*}\right| \leq \alpha(j=1, \cdots, n)$ for all $t \in\left[0, t_{1}\right]$.

Define a function

$$
V(t)=\sum_{i=1}^{n} \int_{x_{i}^{*}}^{x_{i}(t)}\left[g_{i}(\theta)-g_{i}\left(x_{i}^{*}\right)\right] d \theta
$$

for $t \in\left[0, t_{1}\right]$. Obviously, $V(t) \geq 0$.

Similar to the calculation of (3), it is not difficult to see that $(d V(t) / d t) \leq-\sigma V(t)$ for all $t \in\left[0, t_{1}\right]$ and so $V(t) \leq$ $V(0) e^{-\sigma t}$ for all $t \in\left[0, t_{1}\right]$. Then we have

$$
\begin{gathered}
\int_{x_{i}^{*}}^{x_{i}(t)}\left[g_{i}(\theta)-g_{i}\left(x_{i}^{*}\right)\right] d \theta \\
\leq\left\|x(0)-x^{*}\right\| \sqrt{2 k_{i} \sum_{j=1}^{n} k_{j} e^{-(\sigma / 2) t}} \\
(i=1, \cdots, n)
\end{gathered}
$$

for all $t \in\left[0, t_{1}\right]$. 
It follows from Lemma 3 and (5) that

$$
\begin{gathered}
\left|g_{i}\left(x_{i}(t)\right)-g_{i}\left(x_{i}^{*}\right)\right| \\
\leq\left\|x(0)-x^{*}\right\| \sqrt{2 k_{i} \sum_{j=1}^{n} k_{j} e^{-(\sigma / 2) t}} \\
(i=1, \cdots, n)
\end{gathered}
$$

for all $t \in\left[0, t_{1}\right]$. Then from (1) and (6) we have

$$
\begin{aligned}
& D^{+}\left|x_{i}(t)-x_{i}^{*}\right| \\
& \leq-d_{i}\left|x_{i}(t)-x_{i}^{*}\right|+\sum_{j=1}^{n}\left|T_{i j}\right|\left|g_{j}\left(x_{j}(t)\right)-g_{j}\left(x_{j}^{*}\right)\right| \\
& \leq-d_{i}\left|x_{i}(t)-x_{i}^{*}\right|+|| x(0)-x^{*}|| \sum_{j=1}^{n}\left|T_{i j}\right| \\
& \cdot \sqrt{2 k_{j} \sum_{r=1}^{n} k_{r} e^{-(\sigma / 2) t}}
\end{aligned}
$$

for all $t \in\left[0, t_{1}\right]$, and so

$$
\begin{aligned}
&\left|x_{i}\left(t_{1}\right)-x_{i}^{*}\right| \leq|| x(0)-x^{*}||\left[e^{-d_{i} t_{1}}+\frac{2}{2 d_{i}-\sigma} \sum_{j=1}^{n}\left|T_{i j}\right|\right. \\
& \cdot \sqrt{\left.2 k_{j} \sum_{r=1}^{n} k_{r} e^{-(\sigma / 2) t_{1}}\right]} \\
&<\alpha
\end{aligned}
$$

This yields a contradiction and it shows that $\left(x_{1}(t), \cdots, x_{n}(t)\right)^{T}$ stays in $D$ and never cross the boundary of $D$ for all $t \geq 0$.

Since $\left(x_{1}(t), \cdots, x_{n}(t)\right)^{T} \in D$ for all $t \geq 0$, then by Lemma 3,

$$
\begin{aligned}
\int_{x_{i}^{*}}^{x_{i}(t)} & {\left[g_{i}(\theta)-g_{i}\left(x_{i}^{*}\right)\right] d \theta } \\
\geq & \frac{1}{2 k_{i}}\left[g_{i}\left(x_{i}(t)\right)-g_{i}\left(x_{i}^{*}\right)^{2} \quad(i=1, \cdots, n)\right.
\end{aligned}
$$

for all $t \geq 0$. Using (7) and by a slight modification of the proof of Theorem 1 the remains of the proof of this theorem could be derived easily and the details are omitted.

Theorem 5 says that the solutions starting from the open set $Q$ will exponentially converge to the equilibrium in $Q$. Hence, $Q$ is a domain of attraction of the equilibrium.

Theorem 6: Suppose that $D^{+} g_{i}(s)(i=1, \cdots, n)$ are continuous for all $s \in R$. Let $\left(x_{1}^{*}, \cdots, x_{n}^{*}\right)^{T}$ be equilibrium of (1). If there is a small $\epsilon>0$ such that the matrix

$$
\left[-\frac{d_{i}}{D^{+} g_{i}\left(x_{i}^{*}\right)+\epsilon} \delta_{i j}+\frac{T_{i j}+T_{j i}}{2}\right]_{n \times n}, \quad \delta_{i j}= \begin{cases}1, & i=j \\ 0, & i \neq j\end{cases}
$$

is negative definite, then the equilibrium $\left(x_{1}^{*}, \cdots, x_{n}^{*}\right)^{T}$ is exponentially stable.
Proof: Let $k_{i}=D^{+} g_{i}\left(x_{i}^{*}\right)+\epsilon,(i=1, \cdots, n)$, then $0 \leq D^{+} g_{i}\left(x_{i}^{*}\right)<k_{i},(i=1, \cdots, n)$. By the continuity of $D^{+} g_{i}(s)(i=1, \cdots, n)$, there must exist a constant $\alpha>0$ such that $0 \leq D^{+} g_{i}\left(x_{i}\right) \leq k_{i},(i=1, \cdots, n)$ for all $\left(x_{1}, \cdots, x_{n}\right)^{T} \in D$, where

$$
D=\left\{\left(x_{1}, \cdots, x_{n}\right)^{T}|| x_{i}-x_{i}^{*} \mid \leq \alpha,(i=1, \cdots, n)\right\} .
$$

Moreover, since the matrix

$$
\left[-\frac{d_{i}}{k_{i}} \delta_{i j}+\frac{T_{i j}+T_{j i}}{2}\right]_{n \times n}
$$

is negative definite, there exists a small $\sigma \in\left(0, \min _{1 \leq i \leq n}\left(d_{i}\right)\right)$ such that the matrix

$$
T_{\sigma}=\left[-\frac{d_{i}-\sigma}{k_{i}} \delta_{i j}+\frac{T_{i j}+T_{j i}}{2}\right]_{n \times n}, \quad \delta_{i j}= \begin{cases}1, & i=j \\ 0, & i \neq j\end{cases}
$$

is negative semidefinite. The result now follows from Theorem 1 and the proof is completed.

Corollary: Suppose $D^{+} g_{i}(s)(i=1, \cdots, n)$ are continuous for all $s \in R$ with $D^{+} g_{i}\left(x_{i}^{*}\right)>0(i=1, \cdots, n)$ and $\left(x_{1}^{*}, \cdots, x_{n}^{*}\right)^{T}$ is equilibrium of (1). If the matrix

$$
\left[-\frac{d_{i}}{D^{+} g_{i}\left(x_{i}^{*}\right)} \delta_{i j}+\frac{T_{i j}+T_{j i}}{2}\right]_{n \times n}, \quad \delta_{i j}= \begin{cases}1, & i=j \\ 0, & i \neq j\end{cases}
$$

is negative definite, then the equilibrium $\left(x_{1}^{*}, \cdots, x_{n}^{*}\right)^{T}$ is exponentially stable.

Proof: Since the matrix

$$
\left[-\frac{d_{i}}{D^{+} g_{i}\left(x_{i}^{*}\right)} \delta_{i j}+\frac{T_{i j}+T_{j i}}{2}\right]_{n \times n}
$$

is negative definite it implies that there is a small $\epsilon>0$ such that the matrix

$$
\left[-\frac{d_{i}}{D^{+} g_{i}\left(x_{i}^{*}\right)+\epsilon} \delta_{i j}+\frac{T_{i j}+T_{j i}}{2}\right]_{n \times n}
$$

is negative definite, then Theorem 6 is applied. The proof is completed.

\section{EXAMPLES}

Example 1: We are going to design a two-dimensional cellular neural network in the form as follows:

$$
\begin{aligned}
& \frac{d x_{1}(t)}{d t}=-x_{1}(t)+T_{11} f\left(x_{1}(t)\right)+T_{12} f\left(x_{2}(t)\right)+I_{1} \\
& \left.\frac{d x_{2}(t)}{d t}=-x_{2}(t)+T_{21} f\left(x_{1}(t)\right)+T_{22} f\left(x_{2}\right)\right)+I_{2}
\end{aligned}
$$

so that the neural network has a unique equilibrium and this equilibrium is globally exponentially stable with exponential convergence rate at least $\frac{1}{3}$. Where $T_{i j}(i=1, j=1,2)$ and $I_{i}(i=1,2)$ are constants and $f(s)=\frac{1}{2}[|s+1|-|s-1|]$. Obviously, we have $0 \leq D^{+} f(x) \leq 1$. By Theorem 1, it is sufficient to take $\sigma=\frac{2}{3}$ and choose suitable constants $T_{i j}(i, j=1,2)$ such that the matrix

$$
T_{2 / 3}=\left[\begin{array}{cc}
\frac{2}{3}-1+T_{11} & \frac{T_{12}+T_{21}}{2} \\
\frac{T_{12}+T_{21}}{2} & \frac{2}{3}-1+T_{22}
\end{array}\right]
$$


is negative semidefinite. For example, taking $T_{11}=\frac{1}{6}, T_{12}=$ $\frac{1}{2}, T_{21}=\frac{1}{6}, T_{22}=-\frac{1}{3}$, then the matrix $T_{2 / 3}$ is negative semidefinite. It follows from Theorem 1 that $(8)$ has a unique equilibrium $\left(x_{1}^{*}, x_{2}^{*}\right)$ and for any point $\left(x_{1}(0), x_{2}(0)\right) \in R^{2}$ the solution $\left(x_{1}(t), x_{2}(t)\right)$ of (8) starting from this point satisfies

$$
\begin{aligned}
\left|x_{1}(t)-x_{1}^{*}\right| & \leq\left\|x(0)-x^{*}\right\|\left(e^{-t}+2 e^{-(t / 3)}\right) \\
& \leq 3\left\|x(0)-x^{*}\right\| e^{-(t / 3)} \\
\left|x_{2}(t)-x_{2}^{*}\right| & \leq\left\|x(0)-x^{*}\right\|\left(e^{-t}+\frac{3}{2} e^{-(t / 3)}\right) \\
& \leq \frac{5}{2}\left\|x(0)-x^{*}\right\| e^{-(t / 3)}
\end{aligned}
$$

for all $t \geq 0$. This completes the design.

Example 2: Consider the following Hopfield neural network

$$
\begin{aligned}
& \frac{d x_{1}}{d t}=-x_{1}+\frac{17 \ln 4}{15} g\left(x_{2}\right) \\
& \frac{d x_{2}}{d t}=-x_{2}+\frac{17 \ln 4}{15} g\left(x_{1}\right)
\end{aligned}
$$

where $g(s)=\tanh (s)$. Obviously, $D^{+} g(s)=1-g(s)^{2}$ is continuous. It is easy to see that (9) has three equilibrium points $(0,0),(\ln 4, \ln 4)$, and $(-\ln 4,-\ln 4)$. By the Corollary of Theorem 6, it is not difficult to check that the equilibria (ln $4, \ln 4)$ and $(-\ln 4,-\ln 4)$ are locally exponentially stable. Next, we will use the Theorem 5 to calculate an exponential convergence rate and a domain of attraction.

Let

$$
\begin{aligned}
D_{1}= & \left\{\left(x_{1}, x_{2}\right)^{T}|| x_{i}-\ln 4 \mid \leq \ln 4(3-2 \sqrt{2})\right. \\
& \cdot(3+2 \sqrt{2}),(i=1,2)\}
\end{aligned}
$$

and

$$
\begin{aligned}
D_{2}= & \left\{\left(x_{1}, x_{2}\right)^{T}|| x_{i}+\ln 4 \mid \leq \ln 4(3-2 \sqrt{2})\right. \\
& \cdot(3+2 \sqrt{2}),(i=1,2)\} .
\end{aligned}
$$

Obviously, $(\ln 4, \ln 4) \in D_{1}$ and $(-\ln 4,-\ln 4) \in D_{2}$. Since $d g(s) / d s \leq 1 / 2$ for all $|s| \leq \ln \sqrt{3+2 \sqrt{2}}$, we have

$$
\frac{d g\left(x_{1}\right)}{d x_{1}} \leq \frac{1}{2}, \quad \frac{d g\left(x_{2}\right)}{d x_{2}} \leq \frac{1}{2}
$$

for all $\left(x_{1}, x_{2}\right) \in D_{1}$ or $\left(x_{1}, x_{2}\right) \in D_{2}$. Let $k=\frac{1}{2}$, according to the Theorem 5 we should choose a constant $\sigma \in(0,1)$ such that the matrix

$$
T_{\sigma}=\left[\begin{array}{cc}
-2(1-\sigma) & \frac{17 \ln 4}{15} \\
\frac{17 \ln 4}{15} & -2(1-\sigma)
\end{array}\right]
$$

is negative semidefinite. It is easy to see that if $\sigma=1-$ $(17 \ln 4 / 30)$, then

$$
T_{\sigma}=\frac{17 \ln 4}{15}\left[\begin{array}{rr}
-1 & 1 \\
1 & -1
\end{array}\right]
$$

is negative semidefinite. It follows from Theorem 5 that for any $\left(x_{1}(0), x_{2}(0)\right) \in Q_{1}$, where

$$
\begin{aligned}
Q_{1}= & \left\{\left(x_{1}, x_{4}\right)^{T}|| x_{i}-\ln 4 \mid<\frac{30+17 \ln 4}{30+85 \ln 4}\right. \\
& \cdot \ln 4(3-2 \sqrt{2})(3+2 \sqrt{2}),(i=1,2)\} \subset D_{1}
\end{aligned}
$$

the solution $\left(x_{1}(t), x_{2}(t)\right)$ of (9) starting from this point satisfies

$$
\begin{aligned}
\left|x_{1}(t)-\ln 4\right| \leq & \max \left(\left|x_{1}(0)-\ln 4\right|,\left|x_{1}(0)-\ln 4\right|\right) \\
& \cdot\left(e^{-t}+\frac{68 \ln 4}{30+17 \ln 4} e^{-(\sigma / 2) t}\right) \\
\left|x_{2}(t)-\ln 4\right| \leq & \max \left(\left|x_{1}(0)-\ln 4\right|,\left|x_{1}(0)-\ln 4\right|\right) \\
& \cdot\left(e^{-t}+\frac{68 \ln 4}{30+17 \ln 4} e^{-(\sigma / 2) t}\right)
\end{aligned}
$$

for all $t \geq 0$.

Similarly, to the equilibrium $(-\ln 4,-\ln 4) \in D_{2}$, for any point $\left(x_{1}(0), x_{2}(0)\right) \in Q_{2}$, where

$$
\begin{aligned}
Q_{2}= & \left\{\left(x_{1}, x_{2}\right)^{T}|| x_{i}+\ln 4 \mid<\frac{30+17 \ln 4}{30+85 \ln 4}\right. \\
& \cdot \ln 4(3-2 \sqrt{2})(3+2 \sqrt{2}),(i=1,2)\} \subset D_{2}
\end{aligned}
$$

the solution $\left(x_{1}(t), x_{2}(t)\right)$ of (9) starting from this point satisfies

$$
\begin{aligned}
\left|x_{1}(t)+\ln 4\right| \leq & \max \left(\left|x_{1}(0)+\ln 4\right|,\left|x_{1}(0)+\ln 4\right|\right) \\
& \cdot\left(e^{-t}+\frac{68 \ln 4}{30+17 \ln 4} e^{-(\sigma / 2) t}\right) \\
\left|x_{2}(t)+\ln 4\right| \leq & \max \left(\left|x_{1}(0)+\ln 4\right|,\left|x_{1}(0)+\ln 4\right|\right) \\
& \cdot\left(e^{-t}+\frac{68 \ln 4}{30+17 \ln 4} e^{-(\sigma / 2) t}\right)
\end{aligned}
$$

all $t \geq 0$

\section{CONCLUSIONS}

We have studied in this paper a class of neural network that includes the Hopfield neural networks and cellular neural networks. Results for the estimate of exponential convergence rate and exponential stability are established both in local and global situations. These results may be used to design neural networks with desirable exponential convergence rate. Through out this paper we require that the Dini derivative of the neuron input output activations to be bounded. If we drop this condition, the analysis for the exponential stability may become more difficult under the conditions of the connection matrix that we given in this paper. Some of these results could be found in [11] and [12].

\section{REFERENCES}

[1] A. Bouzerdoum and T. R. Pattison, "Neural network for quadratic optimization with bound constraints," IEEE Trans. Neural Networks, vol. 4, pp. 293-303, 1993.

[2] L. O. Chua and L. Yang, "Cellular neural networks: Theory," IEEE Trans. Circuits Syst., vol. 35, pp. 1257-1272, 1988.

[3] M. Forti, "On global asymptotic stability of a class of nonlinear systems arising in neural networks theory," J. Differential Equations, vol. 113, no. 1, pp. 246-164, 1994.

[4] M. Forti, S. Manetti, and M. Marini, "Necessary and sufficient conditions for absolute stability of neural networks," IEEE Trans. Circuits Syst. I, vol. 41, pp. 491-494, 1994. 
[5] M. Forti and A. Tesi, "New conditions for global stability of neural networks with application to linear and quadratic programming problems," IEEE Trans. Circuits Syst. I, vol. 42, pp. 354-366, 1995.

[6] X. B. Liang and L. D. Wu, Comments on "New conditions for global stability of neural networks with application to linear and quadratic programming problems," IEEE Trans. Circuits Syst. I, vol. 44, pp. 1099-1101, 1997.

[7] A. Michel, J. A. Farrell, and W. Porod, "Qualitative analysis of neural networks,” IEEE Trans. Circuits Syst., vol. 36, pp. 229-243, 1989.

[8] S. I. Sudharsanan and M. K. Sundareshan, "Exponential stability and a systematic synthesis of a neural network for quadratic minimization," Neural Networks, vol. 4, pp. 599-613, 1991.
[9] D. W. Tank and J. J. Hopfield, "Simple neural optimization networks: An A/D converter, signal decision circuit, and a linear programming circuit," IEEE Trans. Circuits Syst., vol. 33, pp. 533-541, 1986.

[10] H. Yang and T. S. Dillon, "Exponential stability and oscillation of Hopfield graded response neural network," IEEE Trans. Neural Networks, vol. 5, pp. 719-729, 1994

[11] Y. Zhang, "Global exponential stability and periodic solutions of delay Hopfield neural networks," Int. J. Syst. Sci., vol. 27, no. 2, pp. 227-231, 1996.

[12] Y. Zhang, S. M. Zhong, and Z. L. Li, "Periodic solutions and global stability of delay Hopfield neural networks," Int. J. Syst. Sci., vol. 27, no. 9, pp. 895-901, 1996. 